\title{
UNIQUE BEST NONLINEAR APPROXIMATION IN HILBERT SPACES
}

\author{
CHARLES K. CHUI AND PHILIP W. SMITH
}

ABSTRACT. Using the notion of curvature of a manifold, developed by J. R. Rice and recently studied by E. R. Rozema and the second named author, the authors prove the following result: Let $H$ be a Hilbert space and $F$ map $R^{n}$ into $H$ such that $F$ is a homeomorphism onto $\mathcal{F}=F\left(R^{n}\right)$ and is twice continuously Fréchet differentiable. Then if $F^{\prime}(a) \cdot R^{n}$ is of dimension $n$ for all $a \in R^{n}$, the manifold $\mathcal{F}$ has finite curvature everywhere. It follows that there is a neighborhood $\mathcal{U}$ of $\mathcal{F}$ such that each $u \epsilon$ $\mathcal{U}$ has a unique best approximation from $\mathcal{F}$. However, these results do not hold in general for uniformly smooth Banach spaces.

1. Introduction. Nonlinear approximation arises naturally in many problems. In particular, where algorithmic solutions are desired, uniqueness questions are of great concern. Many recent papers have been written on this subject; for example, see [3], [4], [5], [6], and [7]. In [4], Rozema and the second named author have studied the question of unique best approximation in uniformly smooth Banach spaces. This note is a completion of [4] in the sense that for a Hilbert space it gives a sufficient condition for finite curvature, and hence unique best approximation, in terms of smoothness of the map.

Let $F$ map $R^{n}$ into a Hilbert space $H$. We will denote by $\mathcal{F}$ the image of $R^{n}$ under $F$ and by $F^{\prime}(\alpha)$ the Fréchet derivative of $F$ at $a \in R^{n}$. Throughout this paper we will assume that $F$ satisfies the following three conditions:

(a) $F$ is a homeomorphism onto its image $\mathcal{F}$ (where $\mathcal{F}$ is given the topology induced by $H$ ).

(b) The first and second Fréchet derivatives of $F$ exist and are continuous. (We will denote the second Fréchet derivative of $F$ at $\alpha$ by $F^{\prime \prime}(\alpha)$.)

(c) The dimension of $F^{\prime}(\alpha) \cdot R^{n}$ is $n$ for each $\alpha \in R^{n}$.

As in [4], we define the tangent plane $T(x)$ to $\mathcal{F}$ at $x=F(\alpha)$ by $T(x)$ $=x+F^{\prime}(\alpha) \cdot R^{n}$, and the set of normals $\Re(x)$ to $\mathcal{F}$ at $x$ by $\Re(x)=$

Received by the editors February 6, 1974.

AMS (MOS) subject classifications (1970). Primary $41 \mathrm{A50}$.

Key words and phrases. Nonlinear approximation, curvature, unique best approximation, splines, Hilbert space. 
$\{y:(y-x) \perp T(x)\}$. Let $x$ and $z$ be in $\mathcal{F}$, and for $y \in \Re(x)$, we set

$$
\rho(x, y, z)=\inf \|y(z)-z\|
$$

where the infimum is taken over all the $y(z)$ that lie on the line generated by $x$ and $y$ and satisfy $\|y(z)-x\|=\|y(z)-z\|$. If no $y(z)$ exists, we set $\rho(x, y, z)=\infty$. Let

$$
\rho(x, y)=\lim _{z \rightarrow x, z \in \mathcal{F}} \inf _{z} \rho(x, y, z) .
$$

The radius of curvature $\rho(x)$ of $\mathfrak{F}$ at $x$ is defined in [4] as

$$
\rho(x)=\inf _{y \in \pi(x)} \rho(x, y)
$$

and the curvature $\sigma(x)$ of $\mathcal{F}$ at $x$ is defined by $\sigma(x)=1 / \rho(x)$. For simplicity we will only consider real Hilbert spaces.

2. Finite curvature in terms of smoothness. We first establish the following theorem for a Hilbert space $H$. In the next section we will see that this result does not necessarily hold for a uniformly smooth Banach space in general.

Theorem 1. Let $F: R^{n} \rightarrow H$ satisfy (a), (b), and (c). Then the manifold $\mathfrak{F}$ has finite curvature everywhere.

Proof. We first calculate $\rho(x, y, z)$ where $x=F(\alpha)$ and $z=F(\beta)$ with $\alpha, \beta \in R^{n}$, and $y$ lies on $\Re(x)$. We pick the element $y(z) \in \Re(x)$ where $y(z)=x+t(y-x), t \in R^{1}$, so that $\|y(z)-x\|=\|y(z)-z\|$. Hence,

$$
\|x\|^{2}-2\langle x+t(y-x), x\rangle=\|z\|^{2}-2\langle x+t(y-x), z\rangle .
$$

Solving for $t$, we obtain

$$
t=\frac{\|z\|^{2}+\|x\|^{2}-2\langle x, z\rangle}{2\langle y-x, z-x\rangle}=\frac{\|z-x\|^{2}}{2\langle y-x, z-x\rangle},
$$

and

$$
\rho(x, y, z)=\|y(z)-z\|=\|y(z)-x\|=\frac{\|z-x\|^{2}\|y-x\|}{2|\langle y-x, z-x\rangle|} .
$$

Fix $x \in \mathcal{F}$. To show that $\mathcal{F}$ has finite curvature at $x$, we will bound $\rho(x, y, z)$ from below. Subtracting and adding $\left\langle y-x, F^{\prime}(\alpha) \cdot(\beta-\alpha)\right\rangle$ in the denominator of $\rho(x, y, z)$ yields

$$
\langle y-x, z-x\rangle=\left\langle y-x,(z-x)-F^{\prime}(\alpha) \cdot(\beta-\alpha)\right\rangle
$$


since $y$ is in $\Re(x)$. Using the Schwarz inequality, we have

$$
|\langle y-x, z-x\rangle| \leq\|y-x\|\left\|(z-x)-F^{\prime}(\alpha) \cdot(\beta-\alpha)\right\| \text {. }
$$

Hence,

$$
\rho(x, y, z) \geq \frac{\|z-x\|^{2}}{2\left\|(z-x)-F^{\prime}(\alpha) \cdot(\beta-\alpha)\right\|} .
$$

Next, we want to find an upper bound for the above denominator when $z$ is close to $x$. Since $F$ is twice continuously Fréchet differentiable in a neighborhood of $a$, we have (cf. [2, pp. 99, 180])

$$
\begin{aligned}
& \left\|F(\beta)-F(\alpha)-F^{\prime}(\alpha) \cdot(\beta-\alpha)\right\| \\
& \quad=\left\|\left[\int_{0}^{1}(1-s) F^{\prime \prime}(\alpha+s(\beta-\alpha)) d s\right] \cdot(\beta-\alpha)^{(2)}\right\| \leq c_{1}|\beta-\alpha|^{2}
\end{aligned}
$$

for some positive constant $c_{1}$ independent of $\beta$ if $\beta$ is close to $\alpha$. Here, $(\beta-\alpha)^{(2)}$ stands for $((\beta-\alpha),(\beta-\alpha))$ and $|\cdot|$ is a norm in $R^{n}$. This gives, for $\beta$ close to $\alpha$ (or $z$ close to $x$ ),

$$
\rho(x, y, z) \geq \frac{\|F(\beta)-F(\alpha)\|^{2}}{2 c_{1}|\beta-\alpha|^{2}} .
$$

It is known (cf. [1, p. 219], [4]) that

$$
F(\beta)-F(\alpha)=\lim _{i \rightarrow \infty}\left(\sum_{j=1}^{i} t_{i j} F^{\prime}\left(\gamma_{i j}\right)\right) \cdot(\beta-\alpha)
$$

when $t_{j i} \geq 0, \sum_{j=1}^{j} t_{i j}=1$, and $\gamma_{i j}$ lies on the line segment formed by $\alpha$ and $\beta, 1 \leq j \leq i, i=1,2, \cdots$. By assumption (c), we see that there is a positive constant $c_{2}$ independent of $\beta$ so that

$$
\left\|F^{\prime}(\alpha) \cdot(\beta-\alpha)\right\| \geq c_{2}|\beta-\alpha|
$$

for all $\beta$. From the continuity of $F^{\prime}$, it follows that when $\beta$ is close to $\alpha$,

$$
\left\|\lim _{i \rightarrow \infty} \sum_{j=1}^{i} t_{i j} F^{\prime}\left(\gamma_{i j}\right)-F^{\prime}(\alpha)\right\| \leq c_{2} / 2
$$

Thus, for $\beta$ close to $\alpha$,

$$
-\left\|\lim _{i \rightarrow \infty}\left\{\sum_{j=1}^{i} t_{i j} F^{\prime}\left(\gamma_{i j}\right)\right\} \cdot(\beta-\alpha)\right\|+\left\|F^{\prime}(\alpha) \cdot(\beta-\alpha)\right\| \leq 1 / 2 c_{2}|\beta-\alpha|,
$$


or

$$
1 / 2 c_{2}|\beta-\alpha| \leq \lim _{i \rightarrow \infty}\left\|\sum_{j=1}^{i} t_{i j} F^{\prime}\left(\gamma_{i j}\right) \cdot(\beta-\alpha)\right\|=\|F(\beta)-F(\alpha)\|,
$$

so that

$$
\rho(x, y, z) \geq \frac{c_{2}|\beta-\alpha|^{2}}{2 c_{1}|\beta-\alpha|^{2}}=c_{3}>0
$$

for all $z=F(\beta)$ close to $x=F(\alpha)$. Hence,

$$
\rho(x, y)=\lim _{z \rightarrow x, z \in \mathcal{F}} \inf _{x} \rho(x, y, z) \geq c_{3}
$$

and $\rho(x)=\inf _{y \in \pi(x)} \rho(x, y) \geq c_{3}$, or $\sigma(x) \leq 1 / c_{3}<\infty$. This completes the proof of the theorem.

3. Unique and nonunique best approximation, and an application. As an immediate consequence of Theorem 1 above and Theorem 4.1 in [4], we have the following:

Theorem 2. Let $F: R^{n} \rightarrow H$ satisfy (a), (b), and (c). There is a neighborhood $\mathcal{U}$ of $\mathcal{F}$ such that each $u \in \mathcal{U}$ has a unique best approximation from $\mathcal{F}$.

Theorems 1 and 2 could have been stated in terms of "pieces" of manifolds in the following way. Let $\Omega$ be an open subset of $R^{n}$ and let $F: \Omega \rightarrow$ $H$ satisfy (a), (b), and (c) with the exception that (c) is modified to (c') by

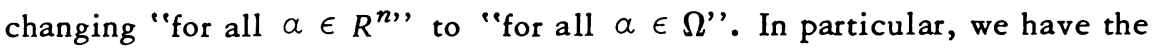
following

Corollary. Let $\Omega$ be an open subset of $R^{n}$ and let $F: \Omega \rightarrow H$ satisfy (a), (b), and ( $\left.\mathrm{c}^{\prime}\right)$. Then $F(\Omega)=\mathcal{F}$ has a neighborhood $\mathcal{U}$ so that every $u \in \mathcal{U}$ has a unique best approximation in $\mathcal{F}$.

The proof of this corollary requires no new idea and will be omitted. As an example, consider the spline mapping $F: R^{2 N+n+1} \rightarrow L^{2}[0,1]$ defined by

$$
F(\alpha)=F\left(a_{0}, \cdots, a_{2 N+n}\right)=\sum_{j=0}^{n} a_{j} t^{j}+\sum_{j=1}^{N} a_{n+j}\left(t-a_{N+n+j}\right)_{+}^{n}
$$

where for a real number $t,(t)_{+}=\max (t, 0)$. Let $\Omega_{1}=\left\{\alpha \in R^{2 N+n+1}: 0<\right.$ $\alpha_{n+N+1}<\cdots<\alpha_{n+2 N}<1, \alpha_{j} \neq 0$ for $\left.n+1 \leq j \leq n+N\right\}$ and $\Omega=\left\{\alpha \in \Omega_{1}\right.$ : 
$|\alpha|<r<\infty\}$. Clearly, $F: \Omega \rightarrow L^{2}[0,1]$ satisfies (a), (b), and (c') if $n \geq 3$. Indeed, by considering $a \in \Omega_{1}$, we can guarantee that ( $c$ ') holds, and in order to guarantee that (a) holds, we must bound $\alpha \in \Omega_{1}$, and hence, we restrict ourselves to $\Omega$. Finally, for $n \geq 3$, it is clear that (b) holds. Thus the corollary applies and we may conclude that $\mathcal{F}=F(\Omega)$ has a neighborhood $\mathcal{U}$ of uniqueness. That is, every $L^{2}[0,1]$ function in $U$ has a unique spline approximation in $L^{2}[0,1]$ from $\mathcal{F}$. The reader can see from the method of proof that in this example the constants $c_{1}$ and $c_{2}$, which determine $\mathcal{U}$, can be estimated.

These results are in some sense surprising since they are not true in general when the range of $F$ is just a uniformly smooth Banach space. For example, let $l^{p}(2), 1 \leq p<\infty$, be the two-dimensional space $R^{2}$ with the norm $\|(x, y)\|=\left(|x|^{p}+|y|^{p}\right)^{1 / p}$ and let $F: R^{1} \rightarrow l^{p}(2)$ satisfy (a), (b), and (c) with $F(t)=(\cos t$, sin $t)$ in a neighborhood $\Omega$ of $t=0$. Then $F$ is clearly infinitely Fréchet differentiable in $\Omega$. If $p>2$ and $\epsilon>0$ is small enough, it is easy to see that the points $(s, 0)$ with $1-\epsilon<s<1$ do not have unique best approximations from $\mathcal{F}=F(\Omega)$. Since finite curvature implies the existence of a neighborhood of uniqueness, we can conclude that $\mathcal{F}$ has infinite curvature at $t=0$.

\section{REFERENCES}

1. V. I. Averbuh and O. G. Smoljanov, Differentiation theory in linear topological spaces, Uspehi Mat. Nauk 22 (1967), no. 6 (138), 201-260 = Russian Math. Surveys 22 ( 1967), no. 6, 201-258. MR 36 \#6933.

2. J. Dieudonné, Foundations of modern analysis, Pure and Appl. Math., vol. 10, Academic Press, New York, 1960. MR 22 \#11074.

3. J. R. Rice, The approximation of functions. Vol. II. Nonlinear and multivariate theory, Addison-Wesley, Reading, Mass., 1969. MR 39 \#5989.

4. E. R. Rozema and P. W. Smith, Nonlinear approximation in uniformly smooth Banach spaces, Trans. Amer. Math. Soc. 188 (1974), 199-212.

5. I. Singer, Best approximation in normed vector spaces by elements of vector subspaces, Ed. Acad. Repub. Soc. Roman̂ia, Bucharest, 1967; English Transl., Die Grundlehren der math. Wissenschaften, Band 171, Springer-Verlag, New York and Berlin, 1970. MR 38 \#3677; 42 \#4937.

6. D. E. Wulbert, Uniqueness and differential characterization of approximations from manifolds of functions, Amer. J. Math. 93 (1971), 350-366. MR 45 \#4036.

7. Nonlinear approximation with tangential characterization, Amer. J. Math. 93 ( 1971 ), $718-730$. MR 45 \#4037.

DEPARTMENT OF MATHEMATICS, TEXAS A \& M UNIVERSITY, COLLEGE STATION, TEXAS 77843 